\title{
Dynamic piezoelectric stimulation enhances osteogenic differentiation of human adipose stem cells
}

Clarisse Ribeiro, ${ }^{1,2}$ Jenita Pärssinen, ${ }^{3,4}$ Vítor Sencadas, ${ }^{1,5}$ Vítor Correia, ${ }^{1,2,6} \mathrm{~S}$.

Miettinen, ${ }^{3}$ Vesa P. Hytönen, ${ }^{3,4}$ Senentxu Lanceros-Méndez ${ }^{1,2, *}$

${ }^{1}$ Center/Department of Physics, University of Minho, Braga 4710-057, Portugal

${ }^{2}$ INL - International Iberian Nanotechnology Laboratory, 4715-330 Braga,

Portugal

${ }^{3}$ BioMediTech, University of Tampere, Tampere 33014, Finland

${ }^{4}$ Fimlab Laboratories Ltd., Tampere 33520, Finland

${ }^{5}$ Instituto Politécnico do Cávado e do Ave, Campus do IPCA, Barcelos 4750-810,

Portugal

${ }^{6}$ Algoritmi Research Centre, Universidade do Minho, Campus de Azurém, 4800-058

Guimarães, Portugal.

*Corresponding author. Address: Center/Department of Physics, University of Minho, Braga 4710-057, Portugal. Tel.: +351 253604073.

E-mail address: lanceros@ @isica.uminho.pt 


\begin{abstract}
This work reports on the influence of the substrate polarization of electroactive $\beta$-PVDF on human adipose stem cells (hASCs) differentiation under static and dynamic conditions. hASCs were cultured on different $\beta$-PVDF surfaces (non-poled and "poled ") adsorbed with fibronectin and osteogenic differentiation was determined using a quantitative alkaline phosphatase assay. "Poled -" $\beta$-PVDF samples promote higher osteogenic differentiation, which is even higher under dynamic conditions. It is thus demonstrated that electroactive membranes can provide the necessary electromechanical stimuli for the differentiation of specific cells and therefore will support the design of suitable tissue engineering strategies, such as bone tissue engineering.
\end{abstract}

Keywords: Electroactive polymer, stem cell, osteogenesis, dynamic conditions.

\title{
1. Introduction
}

Tissue engineering and regenerative medicine is a multidisciplinary research field that includes different approaches (cells and/or biomaterial) but typically involves a biocompatible biomaterial, which can be combined with stem cells and different stimuli with the objective of repairing failing organs.

Bone regeneration is one of the most promising investigated therapeutic applications in tissue engineering field. ${ }^{1}$ Human adipose stem cells (hASCs) exhibit large potential for regenerative medicine applications since they are multipotent and have a natural ability to differentiate into different tissues, such as bone, cartilage and fat cells. ${ }^{2}$ In order to induce the differentiation of stem cells to the desired lineage, hASCs require appropriate extracellular stimuli, through chemical (such as growth factors) and physical signals 
(such as mechanical stimulation). ${ }^{3}$ Particularly, those physical stimuli are of great importance since on an in vivo environment cells are continuously subjected to physical stimuli, such as mechanical one, that deeply influence their development. Thus, a mechanical stimulus can increase the activity of specific cells cultured in vitro in a similar way as observed for in vivo environment..$^{4-5}$ In this sense, the use of bioreactors that mimic different stimuli occurring in the body, providing specific biochemical and physical signals can effectively support regulation of cell function. ${ }^{6-7}$

Further, adhesion, proliferation and differentiation of specific cells can be promoted and/or improved by the use of active materials as scaffolds. Recent research has shown the particularly large potential of one class of such active materials: electroactive polymers and, in particular, piezoelectric polymers, as their mechanically varying surface polarization state can influence cell morphology, adhesion, proliferation and differentiation. ${ }^{8}$ This fact is particularly important as many body tissues are subjected to varying electro-mechanical solicitation, such as bone, ${ }^{9}$ which is fact is piezoelectric, similar to observed for collagen (matrix). ${ }^{10-11}$ Thus, varying surface charge can stimulate cell response, ${ }^{7,12}$ and the use of piezoelectric polymers as scaffolds may allow to mimic existing mechanical and electrical signals relevant for biological activity. In this way, electroactive polymers emerged as a novel approach for tissue engineering applications. Poly(vinylidene fluoride) (PVDF) is a biocompatible polymer with the largest piezoelectric response known among polymer materials. ${ }^{13}$ Although many studies have been performed with PVDF, only a few studies have reported its value as biomaterial. Previous works have shown that piezoelectric polymers are able to induce transient surface charge and they have found to induce a higher cell growth and differentiation compared with the non-piezoelectric control materials. ${ }^{7,14}$ It was also demonstrated that when tissue recombinants were cultivated on PVDF, the serum- 
deprived effect could be rescued and submandibular gland tissue recombinant was able to increase epithelial size, synthesize basement membrane and develop new branches without serum. ${ }^{15}$ Additionally, the human neural stem/progenitor cells (hNSCs/NPCs) differentiation on piezoelectric poly(vinylidene fluoride)-trifluoroethylene (PVDFTrFE) fibrous scaffolds has shown that contact guidance combined with the piezoelectric properties promote the neurite extension and neuronal differentiation. ${ }^{16}$ Further, stimulation under dynamic conditions was already tested showing that it can strongly increase cell response, such as cell proliferation. ${ }^{7}$

Considering the high potential of piezoelectric polymers in development of innovative and new "smart" materials, the challenge lies in the exploitation of the electrical stimuli through the mechanical stimulus. It would be important to proof that these stimuli are relevant for tissue engineering strategies.

In this sense, the aim of this work is to study of influence of electro-mechanical stimulus on osteogenic differentiation. Since the absorption of fibronectin helps cell adhesion, ${ }^{17}$ experiments with hASCs cultured on PVDF coated with fibronectin were performed under both static and dynamic conditions, with and without osteogenic medium in order to evaluate the suitability of piezoelectric polymers as smart scaffolds. This work opens new horizons for the use of piezoelectric stimulation in tissue engineering.

\section{Experimental Section}

\subsection{PVDF samples}

PVDF films (110 $\mu \mathrm{m}$ of thickness) were prepared by spreading a solution of PVDF (Solef 1010, Solvay) in N,N-dimethyl formamide (DMF) (20 wt\% PVDF) onto a glass substrate as described previously in ${ }^{17}$. 
The electrical poling of the $\beta$-PVDF films was achieved using a corona discharge inside a home-made chamber at $100{ }^{\circ} \mathrm{C}$. The applied voltage was $10 \mathrm{kV}$ with a constant current of $15 \mu \mathrm{A}$, and the distance between the sample and the tip was $2 \mathrm{~cm}$. Thereafter, the piezoelectric response $(d 33)$ of the poled samples was verified with a wide range $d 33$ meter (model 8000, APC Int. Ltd., Mackeyville, USA). The obtained piezoelectric d33 coefficient was $\sim-32 \mathrm{pC} \mathrm{N}^{-1}$.

The PVDF films used were non-poled $\beta$-PVDF and "poled -" $\beta$-PVDF (cells cultured on the negatively charged side of the material).

\subsection{Samples sterilization}

For the in vitro assays, circular PVDF films were cut with $13 \mathrm{~mm}$ of diameter. The films were sterilized by several immersions in $70 \%$ ethanol for 30 min each. After that, the samples were exposed to ultraviolet light (UV) for $1 \mathrm{~h}$.

\subsection{Fibronectin adsorption}

The fibronectin (FN) coating was performed without and with photoinitiator treatment. The photoinitiator treatment was used in order to introduce covalent coupling between PVDF and deposited fibronectin to ensure, that manipulation of the material does not release the fibronectin. For FN coating (without photoinitiator), FN was adsorbed on the different PVDF films by immersing the material sheets in FN solution with a concentration of $50 \mu \mathrm{g} \mathrm{mL} \mathrm{m}^{-1}$ for $30 \mathrm{~min}$.

For covalent coupling with photoinitiator, a photoinitiator solution (benzophenone in absolute ethanol solution) with a concentration of $10 \mathrm{mg} \mathrm{mL}^{-1}$ was added to the PVDF samples for $5 \mathrm{~min}$ at room temperature. After that, the photoinitiator solution was 
removed and the samples were allowed to dry for $1 \mathrm{~h}$ in laminar flow at room temperature. Then, the FN solution $\left(50 \mu \mathrm{g} \mathrm{mL}^{-1}\right.$ for $\left.30 \mathrm{~min}\right)$ was added to the PVDF samples. Thereafter, the FN solution was removed and the samples were placed in UV irradiator (IBI Ultralinker, Kodak) for $20 \mathrm{~min}$.

For both fibronectin adsorption processes all samples were washed three times with phosphate buffer saline (PBS) solution before cell culture.

\subsection{Cell culture}

The adipose tissue samples were collected in accordance with the Ethics Committee of the Pirkanmaa Hospital District, Tampere, Finland (R03058). hASCs were isolated from subcutaneous adipose tissue samples acquired from a surgical procedure performed at the Department of Plastic Surgery, Tampere University Hospital, Tampere, Finland. The hASCs were isolated from the adipose tissue samples of two different patients using the mechanical and enzymatic method described previously in ${ }^{18}$. hASCs were maintained and expanded in maintenance medium consisting of Dulbecco's modified Eagle's medium/Ham's nutrient mixture F-12 (DMEM/F-12 1:1, Invitrogen) supplemented with 1\% L-alanyl-L-glutamine (GlutaMAX, Invitrogen), 1\% antibiotics (100 $\mathrm{U} \mathrm{mL}^{-1}$ penicillin and $0.1 \mathrm{mg} \mathrm{mL}^{-1}$ streptomycin, Invitrogen), and $10 \%$ allogeneic human serum (HS, PAA Laboratories GmbH.). The experiments were performed at passages 2 to 4 .

Circular PVDF samples were placed in a 24-well tissue culture polystyrene plate and $500 \mu \mathrm{L}$ of cell supension $\left(4 \times 10^{3}\right.$ cell $\mathrm{mL}^{-1}$ ) was added to each well and incubated at $37{ }^{\circ} \mathrm{C}$ in $95 \%$ humidified air containing $5 \% \mathrm{CO}_{2}$. Also, after 7 days of static culture, part of the cell-cultured samples were transferred onto a home-made bioreactor system (dynamic culture). 
The dynamic culture was performed with a mechanical stimulation by placing the culture plate on a vertical vibration module at a frequency of $1 \mathrm{~Hz}$ with maximum amplitude of $\sim 1 \mathrm{~mm}$.

\subsection{Quantification of alkaline phosphatase and DNA}

According to the procedure described in ${ }^{18}$, the in vitro osteogenic differentiation capacity was determined 15 days after the initiation of differentiation using the alkaline phosphatase quantification assay (qALP).

Briefly, the cells were cultured in regular maintenance medium as well as in osteogenic medium (the cell differentiation was started after $24 \mathrm{~h}$ and the maintenance medium was supplemented with $5 \mathrm{nM}$ dexamethasone (Dex, Sigma-Aldrich), $250 \mu \mathrm{M}$ L-ascorbic acid 2-phosphate (AsA2-P, Sigma-Aldrich) and $10 \mathrm{mM} \beta$-glycerophosphate ( $\beta$-GP, Sigma-Aldrich)).

To lyse the cells, the cells were collected after 15 days and placed in $0.1 \%$ of triton buffer solution (Sigma-Aldrich) and frozen at $-70^{\circ} \mathrm{C}$. Afterward, as described in ${ }^{18}$, the amount of p-NP (p-nitrophenol) produced was measured using a microplate reader (BioRad Lab) by recording the absorbance at $405 \mathrm{~nm}$.

To normalize the qALP activity results, the total was quantified from the cell lysate using a CyQUANT Cell Proliferation Assay Kit (Life Technologies Ltd.) in accordance with the manufacturer's protocol. Then, the fluorescence of each sample was measured by exciting the sample at $480 \mathrm{~nm}$ and measuring the emission at $520 \mathrm{~nm}$ using a BioRad Lab reader. 


\subsection{Statistical analysis}

The quantitative results were obtained from triplicate samples. Statistical differences were obtained by ANOVA using Fisher test for the evaluation of the different groups. $\mathrm{P}$ values $<0.005$ were considered to be statistically significant. The results are expressed as the mean \pm SD (standard deviation).

\section{Results and discussion}

In our previous studies, the influence of piezoelectric PVDF films on fibronectin adsorption ${ }^{17}$ and osteogenic differentiation under static conditions ${ }^{18}$ showed that the surface charge of the poled $\beta$-PVDF films influence the conformation of adsorbed fibronectin which modulated the hASCs adhesion on the PVDF films and induced their osteogenic differentiation. It was also verified that the different types of $\beta$-PVDF (nonpoled, "poled +" and "poled -") affect in a different way cell adhesion, proliferation and differentiation and that the influence is dependent on cell type. In particular, investigations with hASCs cultured on PVDF films ${ }^{18}$ has shown that the cell adhesion

on "poled -" $\beta$-PVDF samples is stronger than in cells cultured on the other types of PVDF films. For that reason, "poled -" $\beta$-PVDF was used here to study the potential of piezoelectric stimulation to affect cellular differentiation (Figure 1). Non-poled $\beta$-PVDF was used as control material without piezoelectric function.

Figure 2 shows the hASCs differentiation on different PVDF films determined by the relative qALP expression after 15 days of culture using regular and osteogenic medium under static and dynamic conditions. Regarding static and dynamic conditions, it is verified a higher osteogenic differentiation on "poled -" $\beta$-PVDF in dynamic conditions 
than in static conditions. This behavior was not observed for the non-poled $\beta$-PVDF samples. Although osteogenic medium enhanced differentiation as compared to regular medium in all the conditions studied, piezoelectric stimulation showed further enhancement in differentiation when cells were cultured on "poled -" $\beta$-PVDF but not when cultured on non-poled PVDF. The highest amount of differentiation was obtained by combining piezoelectric stimulation and chemical stimulation, i.e. when cells were cultured on "poled -" $\beta$-PVDF in osteogenic medium and under dynamic conditions.

Control experiment where fibronectin was crosslinked to PVDF by using benzophenone crosslinker was also performed and it was observed virtually identical results as compared to those seen in case of fibronectin physisorption. This finding suggests that fibronectin physisorption to PVDF is tight enough to resist piezoelectric stimulation procedure and the effect cannot be further enhanced by covalent linking of the protein layer. In this context, it is good to mention that fibronectin can be considered as "sticky" protein, and the importance of covalent coupling may vary between proteins.

In conclusion, it is possible to claim that the incorporation of both physical and chemical stimulus in in vitro culture enhances the osteogenic differentiation. More particularly, piezoelectric materials may provide the necessary electrical stimulus for osteogenic differentiation, mimicking the mechanically stimulated environments existing in the body, improving thus bone regeneration strategies.

\section{Conclusions}

This work proves that the use of a suitable combination of physical and biochemical stimuli in a biomimetic approach can lead to the development of more efficient and successful tissue engineering strategies. In particular, the use of piezoelectric stimuli, 
mechanically induced variations of surface charge, can lead to enhanced osteogenic differentiation of human adipose stem cells (hASCs). Therefore, dynamic mechanical conditions in combination of suitable osteogenic differentiation media may offer tools to better mimick the conditions found in vivo.

Acknowledgements: This work is funded by FEDER funds through the "Programa Operacional Fatores de Competitividade - COMPETE" and by national funds arranged by FCT- Fundação para a Ciência e a Tecnologia, project references PTDC/CTMNAN/112574/2009 and PEST-C/FIS/UI607/2014. The authors also thank funding from Matepro -Optimizing Materials and Processes", ref. NORTE-07-0124-FEDER000037", co-funded by the "Programa Operacional Regional do Norte" (ON.2 - O Novo Norte), under the "Quadro de Referência Estratégico Nacional” (QREN), through the "Fundo Europeu de Desenvolvimento Regional" (FEDER). CR, VS and VC thank the FCT for the SFRH/BPD/90870/2012, SFRH/BPD/64958/2009 and SFRH/BPD/97739/2013 grants, respectively. Academy of Finland is acknowledged for research funding (projects $136288(\mathrm{VH})$ and $256931(\mathrm{JP})$ ).

\section{References}

1. Fu RH, Wang YC, Liu SP, Huang CM, Kang YH, Tsai CH, Shyu WC, Lin SZ. Differentiation of Stem Cells: Strategies for Modifying Surface Biomaterials. Cell Transplant 2011; 20: 37-47.

2. Zuk PA, Zhu M, Ashjian P, De Ugarte DA, Huang JI, Mizuno H, Alfonso ZC, Fraser JK, Benhaim P, Hedrick MH. Human adipose tissue is a source of multipotent stem cells. Mol Biol Cell 2002; 13: 4279-4295. 
3. Kilian KA, Bugarija B, Lahn BT, Mrksich M. Geometric cues for directing the differentiation of mesenchymal stem cells. Proceedings of the National Academy of Sciences of the United States of America 2010; 107: 4872-4877.

4. Butler DL, Goldstein SA, Guilak F. Functional tissue engineering: the role of biomechanics. J Biomechanical Eng 2000; 122: 570-575.

5. Altman GH, Horan RL, Martin I, Farhadi J, Stark PRH, Volloch V, Richmond JC, Vunjak-Novakovic G, Kaplan DL. Cell differentiation by mechanical stress. FASEB journal: official publication of the Federation of American Societies for Experimental Biology 2002; 16: 270-272.

6. Alvarez-Barreto JF, Landy B, VanGordon S, Place L, DeAngelis PL, Sikavitsas VI. Enhanced osteoblastic differentiation of mesenchymal stem cells seeded in RGD-functionalized PLLA scaffolds and cultured in a flow perfusion bioreactor. J Tissue Eng Regen Med 2011; 5: 464-475.

7. Ribeiro C, Moreira S, Correia V, Sencadas V, Rocha JG, Gama FM, Ribelles JLG, Lanceros-Mendez S. Enhanced proliferation of pre-osteoblastic cells by dynamic piezoelectric stimulation. Rsc Adv 2012; 2: 11504-11509.

8. Weber N, Lee YS, Shanmugasundaram S, Jaffe M, Arinzeh TL. Characterization and in vitro cytocompatibility of piezoelectric electrospun scaffolds. Acta Biomater 2010; 6: 3550-3556.

9. $\quad$ Fukada E, Yasuda I. On the piezoelectric effect of bone. J Phys Soc Japan 1957; 12: $1158-1162$.

10. Ahn AC, Grodzinsky AJ. Relevance of collagen piezoelectricity to "Wolff's Law": A critical review. Med Eng Phys 2009; 31: 733-741.

11. Fukada E, Ueda H, Rinaldi R. Piezoelectric and related properties of hydrated collagen. Biophys J 1976; 16: 911-918. 
12. Schneider GB, English A, Abraham M, Zaharias R, Stanford C, Keller J. The effect of hydrogel charge density on cell attachment. Biomaterials 2004; 25: 3023-3028.

13. Martins P, Lopes AC, Lanceros-Mendez S. Electroactive phases of poly(vinylidene fluoride): Determination, processing and applications. Prog Polym Sci 2014; 39: 683-706.

14. Valentini RF, Vargo TG, Gardella Jr JA, Aebischer P. Electrically charged polymeric substrates enhance nerve fibre outgrowth In vitro. Biomaterials 1992; 13: 183-190.

15. Yang TL, Hsiao YC, Lin SJ, Lee HW, Lou PJ, Ko JY, Young TH. Biomaterial mediated epithelial-mesenchymal interaction of salivary tissue under serum free condition. Biomaterials 2010; 31: 288-295.

16. Lee Y-S, Arinzeh TL. The influence of piezoelectric scaffolds on neural differentiation of human neural stem/progenitor cells. Tissue Eng A 2012; 18 : 2063-2072.

17. Ribeiro C, Panadero JA, Sencadas V, Lanceros-Mendez S, Tamano MN, Moratal D, Salmeron-Sanchez M, Gomez Ribelles JL. Fibronectin adsorption and cell response on electroactive poly(vinylidene fluoride) films. Biomed Mater 2012; 7: 035004.

18. Pärssinen J, Hammarén H, Rahikainen R, Sencadas V, Ribeiro C, Vanhatupa S, Miettinen S, Lanceros-Méndez S, Hytönen VP. Enhancement of adhesion and promotion of osteogenic differentiation of human adipose stem cells by poled electroactive poly(vinylidene fluoride). J Biomed Mater Res A 2014; doi: 10.1002/jbm.a.35234.

\section{Figures and Figure Captions}




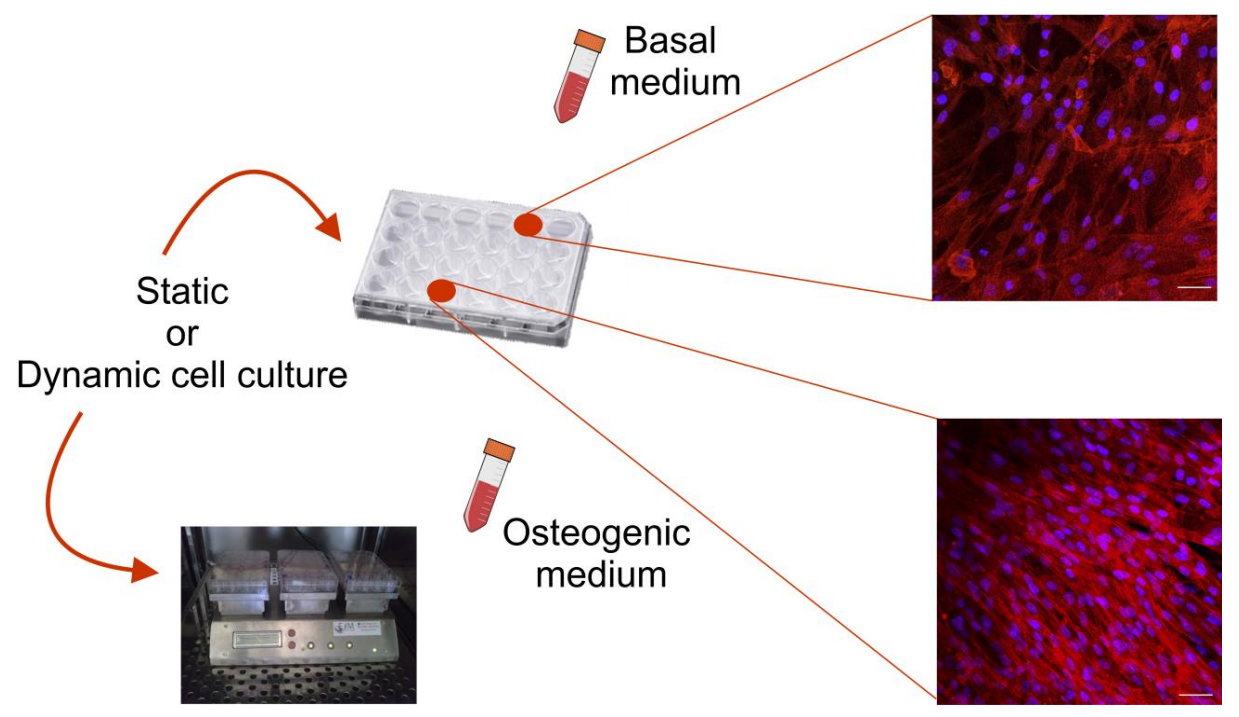

Figure 1 - Scheme of cell culture assay (static and dynamic conditions; basal and osteogenic medium) and representative confocal images of cells cultured 7 days on "poled -" $\beta$-PVDF samples. The confocal images are presented in the same scale (scale bar of $50 \mu \mathrm{m}$ ). 


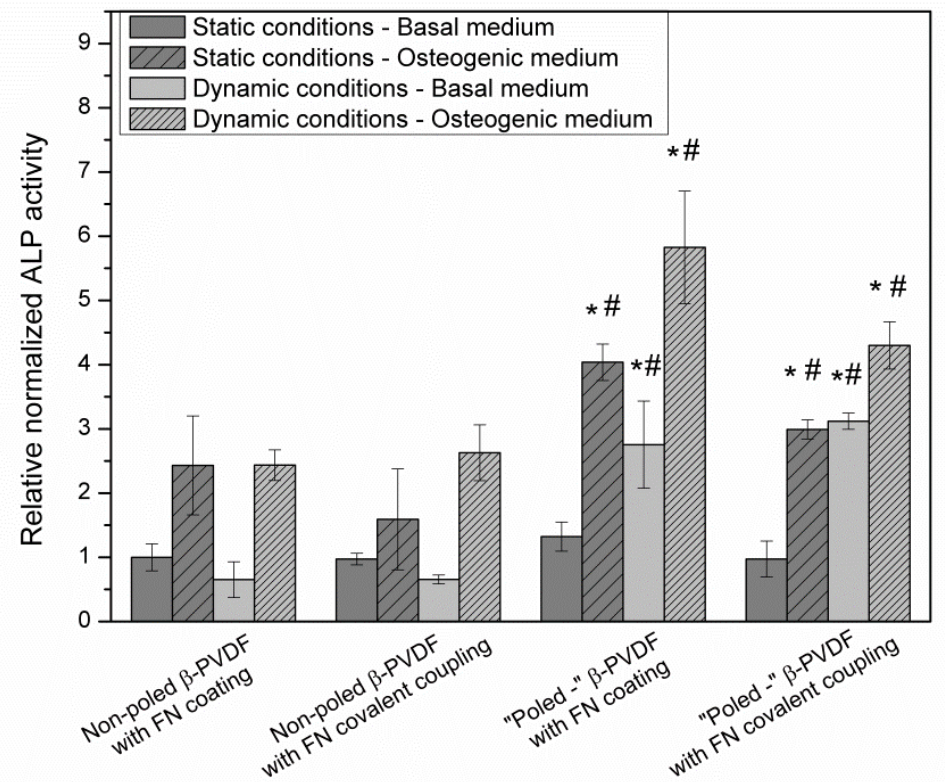

Figure 2 - hASCs differentiation on different PVDF films determined by relative qALP expression after 15 days of culture using regular and osteogenic medium under static and dynamic conditions. The ALP expression was normalized against the DNA content of the cells using the CyQuant cell proliferation assay. * $\mathrm{P} \leq 0.005$ vs non-poled $\beta$-PVDF with FN coating under static conditions and basal medium; \# $\mathrm{P} \leq 0.005$ vs "poled -" $\beta$-PVDF with FN coating under static conditions and basal medium. 